\title{
Modeling and Analysis of a 6-DOF Flexible Hypersonic Flight Vehicle*
}

\author{
Xibin ZHANG, ${ }^{\dagger}$ Zhenxing ZHANG, and Yumei DiNG \\ School of Science, Tianjin University of Science and Technology, Tianjin 300222, China
}

\begin{abstract}
The aerodynamic, structure and propulsion interactions in a six-degree-of-freedom (6-DOF) flexible hypersonic vehicle bring great challenges to vehicle modeling and control design. This paper focus on the modeling of a 6-DOF flexible hypersonic flight vehicle. The flexible dynamic and three-dimensional aerodynamic loads are calculated by using or combining an assumed modes method, variation theory and shock/expansion theory. A control-oriented nonlinear model $(\mathrm{COM})$ is obtained using curve-fitted approximation of the forces and moments on the vehicle. The COM is then linearized about a steady flight condition, and the rigid/flexible dynamics and coupling of the 6-DOF vehicle model with aerodynamic, structure and propulsion interactions are analyzed comprehensively.
\end{abstract}

Key Words: Flexible Hypersonic Flight Vehicle, 6-DOF, Curve-fit, Control-oriented Model, Coupling

\section{Introduction}

The hypersonic flight vehicle has the potential for a wide variety of military and civilian applications. The study on such vehicles has been attracting increased attention in the aerospace field in recent years. The navigation and controller design for hypersonic vehicles is a challenging task due to the complex interactions among the aerodynamics, structural dynamics and propulsion system. ${ }^{1)}$ Building an actual vehicle model that captures the real physical characteristics can provide control engineers a fundamental understanding of the dynamics of the vehicle system at hand. Furthermore, the model can be used to assess the flight control design, and promote development of the guidance and control laws for hypersonic vehicles.

Since the 1990s, researchers have attempted to develop hypersonic vehicle models that can simulate real flight environments. For the two-dimensional flight dynamic vehicle model, Chavez and Schmidt ${ }^{2}$ presented a comprehensive analytical model that includes the effects among the aerodynamics, structural dynamics and propulsion, and only the first flexible mode was considered. Bolender and Doman proposed a first principle model $(\mathrm{FPM})^{3)}$ that contains unsteady effects, ${ }^{4)}$ aero-elastic behavior ${ }^{5)}$ and viscous friction ${ }^{6}$ ) using the shock/expansion theory and piston theory with the X-43A configuration. The FPM includes heave coupling between the rigid and flexible dynamics, and is intractable for control design. ${ }^{7)}$ Therefore, some simplified control-oriented models (COMs) were developed based on the FPM, ${ }^{8-12)}$ and then the COMs were applied to design linear and nonlinear controllers. ${ }^{13-16)}$ Clark et al. ${ }^{17,18)}$ presented an integrated elastic aero-propulsion model of a two-dimensional, airbreathing, generic hypersonic flight vehicle using the combined analytic computational fluid dynamics (CFD) approach. Zeng et al. ${ }^{19)}$ established an aero-servoelastic model

(C) 2020 The Japan Society for Aeronautical and Space Sciences

*Received 5 July 2019; final revision received 25 February 2020; accepted for publication 18 March 2020

†Corresponding author, brooks@tust.edu.cn with rigid-body motion, elastic fuselage model, wing/pivot system models and actuator dynamics using Shock-Expansion theory and the local piston theory.

For the three-dimensional flight dynamic vehicle model, Keshmiri $^{20)}$ established a nonlinear model of the longitudinal and lateral-directional dynamics for a wing-cone, airbreathing hypersonic vehicle. CFD simulations are used to develop a merged aerodynamic database. Zong et al. ${ }^{21)}$ presented an aero-servoelastic model of a six-degrees-of-freedom (6-DOF) hypersonic flight vehicle using mechanism analysis, while excluding the propulsion system. Ye et al. ${ }^{22)}$ analyzed the impact of the aero-thermoelasticity effects at the engine inlet of a three-dimensional hypersonic vehicle using CFD technology. Frendreis et al. ${ }^{23,24)}$ developed a three-dimensional, 6-DOF hypersonic vehicle model that includes the coupling of aerodynamic, structure and propulsion by combining mechanism analysis and CFD technology. Sudalagunta et al. ${ }^{25)}$ presented an aeroelastic control-oriented model of an air-breathing hypersonic vehicle taking into account the interaction among aerodynamic, gravitational, control, inertial and vibrational effects. The high-frequency free vibration modes were included in the model.

Most of the vehicle models are restricted to longitudinal dynamics. However, the accuracy of the two-dimensional model is limited due to the fact that it cannot capture the actual three-dimensional phenomena encountered. ${ }^{23)}$ CFD technology and mechanism analysis are two available tools to establish the vehicle model, where the CFD technology simulates the flight environment, and mechanism analysis reveals insight physical characteristics of the vehicle model. The analysis tools will support designing of the vehicle control systems and studying the three-dimensional hypersonic vehicle.

This paper describes a three-dimensional hypersonic vehicle configuration. The primary objective is to establish a control-oriented 6-DOF hypersonic vehicle model with aerodynamic, structure and propulsion interactions using the mechanism analysis method. The airframe is modeled as a free-free Euler Bernoulli beam, taking into account the flex- 
ible bending of the forebody and aftbody. Shock/Expansion theory is explored to calculate the aerodynamic load on the vehicle surfaces. Knowledge of the aerodynamic load and existing one-dimensional propulsion model are used to determine the total forces and moments that are intractable for model-based control design. The analytical expressions of aerodynamic forces, moment and thrust as computed by mechanism analysis are fitted using the multivariate stepwise regression method. The control-oriented model should retain the inherent rigid/flexible couplings, reduce the complexity of the physics-based model and be better suited for analysis.

\section{Model Descriptions}

The hypersonic vehicle is built using a lightweight material and has a long, wedge-shaped forebody that creates an oblique shock at the front of the aircraft. A scramjet engine is located on the underside the vehicle and has a translating cowl door that maintains the shock-on-lip condition for off-design flight conditions. A side view of the hypersonic vehicle and the internal layout are shown in Figs. 1 and 2, respectively. The total length of the fuselage is $L=30.48 \mathrm{~m}$, and the fuselage is divided into three segments: forebody, engine nacelle and aftbody. Figure 3 presents the top view of the hypersonic vehicle, where the upper surface is an isosceles trapezoid with a height equal to the length of the fuselage. The figure also shows the location of the four control panels located on the tail of the aircraft. As shown in Fig. 1, the lower forebody and aftbody are parts of the scramjet engine. The aerodynamic and propulsion system cannot be separated from each other. Furthermore, the flexible bending of the forebody and aftbody affects the pressure distribution on the aircraft and performance of propulsion system. Thus, the aerodynamics, flexible structure, and propulsion interactions, which should be included in the model, make the modeling and control of the vehicle very challenging.

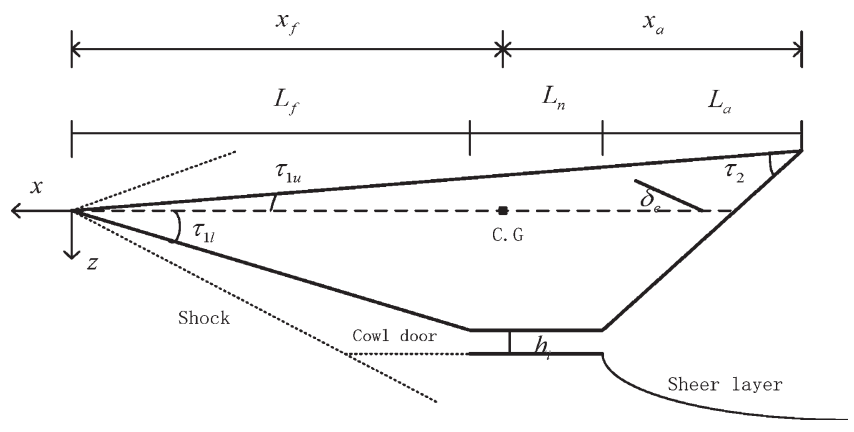

Fig. 1. Side view of the hypersonic vehicle.

\section{Flexible Body Dynamics}

The fuselage is assumed to be a free-free Euler Bernoulli beam. Only the transverse displacements of the beam are of interest, as the main source of fuselage flexibility is longitudinal bending, of which the stiffness is much lower than the torsion and lateral bending. ${ }^{26)}$ The transverse vibration of a free-free beam is governed by the differential equation:

$$
E I \frac{\partial^{4} z(x, t)}{\partial x^{4}}+\bar{m} \frac{\partial^{2} z(x, t)}{\partial t^{2}}=f(x, t)
$$

where, $x$ is the longitudinal coordinate, $t$ is the time, $z(x, t)$ is the vertical coordinate, $E I$ is the stiffness distribution of the beam, $\bar{m}$ is the mass per unit length, and $f(x, t)$ is the force.

Using the principle of separation of variables, the transverse displacement can be expressed as

$$
z(x, t)=\sum_{i=1}^{n} \varphi_{i}(x) \eta_{i}(t),
$$

where, $\varphi_{i}(x)$ is the mode shape of the free-free beam and possess all required derivatives, and $\eta_{i}(t)$ is the generalized modal coordinate.

Substituting Eq. (2) into Eq. (1) and adding the structure damping, the vibration equation (Eq. (1)) can be written as

$$
\ddot{\eta}_{i}+2 \zeta_{i} \omega_{i} \dot{\eta}_{i}+\omega_{i}^{2} \eta_{i}=N_{i}, \quad i=1,2, \ldots, n,
$$

where, $\zeta$ is the structure damping, $\omega_{i}$ is natural frequency which can be obtained by the assumed modes method, ${ }^{7}$ and $N_{i}$ is the generalized force for the $i$ th mode shape of the beam.

Given the loading on the upper and lower body and control surfaces, the generalized forces become

$$
\begin{aligned}
N_{i}= & \int_{0}^{L} \varphi_{i}(x) p_{u p} \mathrm{~d} x-\int_{0}^{L_{f}} \varphi_{i}(x) p_{d} \mathrm{~d} x \\
& -\int_{L_{f}+L_{n}}^{L} \varphi_{i}(x) p_{a} \mathrm{~d} x+\varphi_{i}\left(x_{e}\right) F_{e}+\varphi_{i}\left(x_{r}\right) F_{r},
\end{aligned}
$$

where, $p_{u p}, p_{d}$ and $p_{a}$ are the pressure on the upper surface,

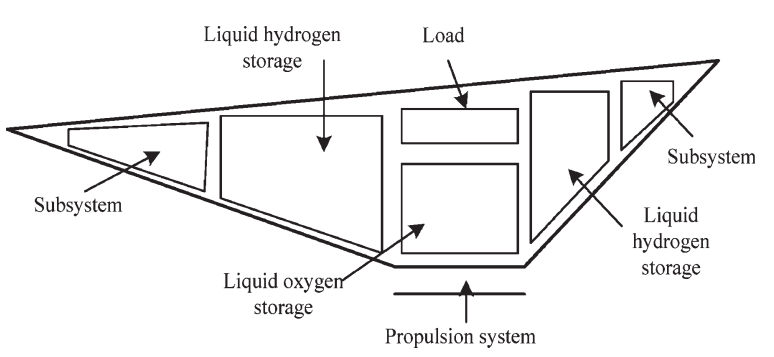

Fig. 2. Internal layout.

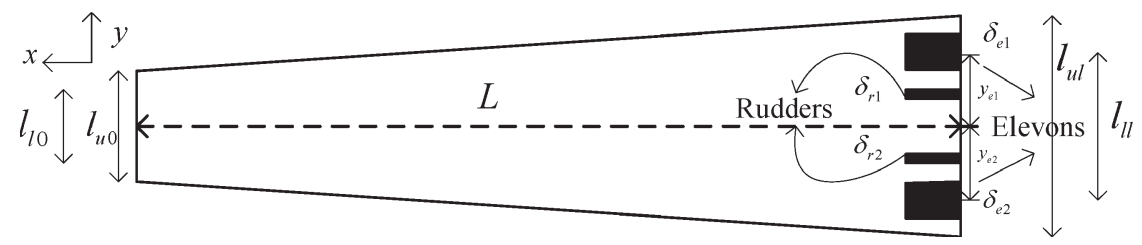

Fig. 3. Top view of the hypersonic vehicle. 
lower forebody and lower aftbody, respectively, and $F_{e}$ and $F_{r}$ are the control surfaces forces.

The vehicle body flexing, however, results in forebody deflection and aftbody deflection, which can be given by

$$
\begin{aligned}
& \Delta \tau_{f}=\left.\sum_{i=1}^{n} \frac{\mathrm{d} \varphi_{i}(x)}{\mathrm{d} x}\right|_{x=0} \eta_{i}(t), \\
& \Delta \tau_{a}=\left.\sum_{i=1}^{n} \frac{\mathrm{d} \varphi_{i}(x)}{\mathrm{d} x}\right|_{x=L} \eta_{i}(t) .
\end{aligned}
$$

Deformation of the vehicle body will directly change the flow characteristics, which will affect the pressure distribution on the surface of the fuselage and the performance of the propulsion system.

\section{Forces and Moments}

We assume that the flow throughout the vehicle body is steady inviscid hypersonic flow, and the aircraft does not turn in to or away from the flow. Therefore, the side slip is neglected. An oblique shock or an expansion fan occurs if the flow is flowing over a concave corner or a convex corner on the vehicle surface. The Oblique Shock Theory or Prandtl-Meyer Expansion Flow Theory ${ }^{3,25)}$ can be used to calculate the pressure on the body, which is dependent upon the angle-of-attack, the vehicle angel and the flexible bending of the forebody and aftbody. Then, we can determine all of the aerodynamic forces and moments acting on the vehicle.

\subsection{Aerodynamics}

The surface of the vehicle body mainly contains four parts: upper surface, lower nacelle, lower forebody and lower aftbody. The surfaces are all isosceles trapezoidal in shape. Similar to the longitudinal dynamics modeling of the vehicle ${ }^{10)}$ we can deduce the forces and resulting moments acting on the upper surface, lower nacelle and lower forebody based on the geometry of the vehicle. The force acting on the lower aftbody is a function of the position of the shear layer that is formed by the interaction of the exhaust with the freestream flow under the vehicle. ${ }^{1,3}$ ) Taking the upper surface of the vehicle body as an example, the body-axes forces and moment are given as

$$
\begin{aligned}
& F_{x, u p}=-p_{u p} S_{u p} \tan \left(\tau_{1 u}-\Delta \tau_{f}\right), \\
& F_{y, u p}=0, \\
& F_{z, u p}=p_{u p} S_{u p}, \\
& M_{x, u p}=z_{u p} F_{x, u p}-x_{u p} F_{z, u p}, \\
& M_{y, u p}=0, \\
& M_{z, u p}=0,
\end{aligned}
$$

where, $S_{u p}$ is the area, $M_{x, u p}, M_{y, u p}$ and $M_{z, u p}$ are the moments, and $\left(x_{u p}, z_{u p}\right)$ is the aerodynamic center of the upper surface relative to the center-of-mass.

\subsection{Control surface forces}

The hypersonic vehicle has four control surfaces: two elevons and two rudders, which are located on the tail of the vehicle as shown in Fig. 3. Oblique Shock Theory or
Prandtl-Meyer Expansion Flow Theory is used to calculate the pressures on the four control surfaces. For the elevons, when the angle of the incidence $\delta=\delta_{e}-\Delta \tau_{a}-\alpha>0$, an oblique shock occurs on the upper surface, and a PrandtlMeyer expansion fan is seen on the lower surface. If $\delta=\delta_{e}-\Delta \tau_{a}-\alpha<0$, an expansion fan forms on top of the elevon and a shock forms on the bottom of the elevon; where, $\delta_{e}$ is the elevon deflection and $\alpha$ is the angle of attack.

For the right rudder, when the angle of incidence is

$i=\frac{\pi}{2}-\arccos \left(\cos \alpha \sin \delta_{r 1}+\sin \alpha \cos \delta_{r 1} \sin \tau_{r 1}\right)>0$,

a shock forms on the inward surface and an expansion fan forms on the outward surface. If

$i=\frac{\pi}{2}-\arccos \left(-\cos \alpha \sin \delta_{r 1}+\sin \alpha \cos \delta_{r 1} \sin \tau_{r 1}\right)>0$,

then an expansion fan forms on the inward surface and a shock forms on the outward surface; where, $\delta_{r 1}$ is the right rudder deflection, and $\tau_{r 1}$ is the inclined angle of the right rudder with respect to the normal vector on the upper-body plane. The same result can be derived for the left rudder.

Then, the control surface forces and moments along the $x, y$ and $z$ directions are given by the following equations

$$
\begin{aligned}
F_{x, c}= & \left(p_{e 1, l}-p_{e 1, u}\right) S_{e 1} \sin \left(\delta_{e 1}-\Delta \tau_{a}\right) \\
& +\left(p_{e 2, l}-p_{e 2, u}\right) S_{e 2} \sin \left(\delta_{e 2}-\Delta \tau_{a}\right) \\
& +\left(p_{r 1, o}-p_{r 1, i}\right) S_{r 1} \cos \delta_{r 1} \\
& -\left(p_{r 2, o}-p_{r 2, i}\right) S_{r 2} \cos \delta_{r 2}, \\
F_{y, c}= & \left(p_{r 1, o}-p_{r 1, i}\right) S_{r 1} \sin \delta_{r 1} \\
& -\left(p_{r 2, o}-p_{r 2, i}\right) S_{r 2} \sin \delta_{r 2}, \\
F_{z, c}= & \left(p_{e 1, l}-p_{e 1, u}\right) S_{e 1} \cos \left(\delta_{e 1}-\Delta \tau_{a}\right) \\
& +\left(p_{e 2, l}-p_{e 2, u}\right) S_{e 2} \cos \left(\delta_{e 2}-\Delta \tau_{a}\right), \\
M_{x, c}= & \left(p_{e 1, l}-p_{e 1, u}\right) S_{e 1} y_{e 1} \cos \left(\delta_{e 1}-\Delta \tau_{a}\right) \\
& -\left(p_{e 2, l}-p_{e 2, u}\right) S_{e 2} y_{e 2} \cos \left(\delta_{e 2}-\Delta \tau_{a}\right), \\
M_{y, c}= & \left(p_{e 1, l}-p_{e 1, u}\right) \\
& \times S_{e 1}\left(x_{e 1} \cos \left(\delta_{e 1}-\Delta \tau_{a}\right)-z_{e 1} \sin \left(\delta_{e 1}-\Delta \tau_{a}\right)\right) \\
& +\left(p_{r 1, o}-p_{r 1, i}\right) S_{r 1} z_{r 1} \cos \delta_{r 1} \\
& -\left(p_{r 2, o}-p_{r 2, i}\right) S_{r 2} z_{r 2} \cos \delta_{r 2} \\
& +\left(p_{e 2, l}-p_{e 2, u}\right) \\
& \times S_{e 2}\left(x_{e 2} \cos \left(\delta_{e 2}-\Delta \tau_{a}\right)-z_{e 2} \sin \left(\delta_{e 2}-\Delta \tau_{a}\right)\right), \\
M_{z, c}= & \left(p_{r 1, o}-p_{r 1, i}\right) S_{r 1} y_{r 1} \sin \delta_{r 1} \\
& -\left(p_{r 2, o}-p_{r 2, i}\right) S_{r 2} y_{r 2} \sin \delta_{r 2},
\end{aligned}
$$

where, $p_{e i, u}$ and $p_{e i, l}$ are the pressure on the $i$ th elevon upper and lower surfaces, respectively, $p_{r i, i}$ and $p_{r i, o}$ are the pressure on the $i$ th rudder inward and outward surfaces, respectively, $S_{e i}$ and $S_{r i}$ are the areas of the control surfaces, and $x_{e i}, y_{e i}, z_{e i}, y_{r i}$ and $z_{r i}$ are the positions of the control surface midpoints, $i=1,2$.

\subsection{Thrust}

The lower forebody and lower aftbody surfaces are part of 
the integrated airframe-engine, and the lower forebody experiences oblique shock. Assuming that the engine is rigid and the forebody flexing reflects the bow shock and air mass flow spillage due to the forebody deflection, then the structure bending influences the engine inlet conditions and pressure distribution in the propulsion system. By applying the momentum theorem, the thrust is given $b^{3)}$ :

$$
T=\dot{m}_{a}\left(V_{e}-V_{\infty}\right)+\left(p_{e}-p_{\infty}\right) \frac{A_{e}}{b}-\left(p_{1}-p_{\infty}\right) \frac{A_{e} / b}{A_{n} A_{d}},
$$

where, $\dot{m}_{a}$ is the air mass flow through the engine, $V_{e}$ is the exit flow velocity, $p_{\infty}$ and $V_{\infty}$ are pressure and velocity of the free-stream flow, respectively, $p_{e}$ is the pressure at the engine exit, $p_{1}$ is the pressure at the inlet, $A_{e} / b$ is the exit area per unit width, and $A_{n}$ is the nozzle area ratio.

\subsection{Body forces and moments}

Once the body forces, thrust and moments have been calculated, we can get the total forces $F_{x}, F_{y}$ and $F_{z}$, and the moments $M_{x}, M_{y}$ and $M_{z}$ in the $x, y$ and $z$ directions, respectively. Then, the forces can be translated into the lift, drag and side force acting on the aircraft using the following coordinate transformation:

$$
\boldsymbol{R}_{\alpha}=\left[\begin{array}{ccc}
\cos \alpha & 0 & \sin \alpha \\
0 & 1 & 0 \\
-\sin \alpha & 0 & \cos \alpha
\end{array}\right] .
$$

The lift, drag and side force are then

$$
\begin{aligned}
& L=F_{x} \sin \alpha-F_{z} \cos \alpha, \\
& D=-F_{x} \cos \alpha-F_{z} \sin \alpha, \\
& Y=F_{y} .
\end{aligned}
$$

The flexible effects are included in force and moment formulations. The body flexing influences the aerodynamic and propulsion system, which results in a vehicle model with strong couplings and highly nonlinear dynamics. If we assume a rigid structure such that $\Delta \tau_{f}=\Delta \tau_{a}=0$ is excluded, then the formulas in Eq. (10) are the steady aerodynamic forces of a rigid body vehicle.

\section{6-DOF Flight Dynamic Equations of Motion}

The 6-DOF model of the hypersonic vehicle can be derived using the Newtonian approach with the flat-earth assumption, ${ }^{27)}$ and it includes the position information, attitude information and flexible state of the aircraft. The 6-DOF equations of motion for a three-dimensional flexible hypersonic vehicle mainly consist of 14 differential equations:

$$
\begin{aligned}
& \dot{x}=V \cos \gamma \cos \chi, \\
& \dot{y}=V \cos \gamma \sin \chi, \\
& \dot{h}=-V \sin \gamma, \\
& \dot{V}=\frac{1}{m}(-D+T \cos \alpha-m g \sin \gamma), \\
& \dot{\chi}=\frac{1}{m V \cos \gamma}(Y \cos \mu+L \sin \mu+T \sin \mu \sin \alpha),
\end{aligned}
$$

$$
\begin{aligned}
\dot{\gamma}= & \frac{1}{m V}(L \cos \mu+T \cos \mu \sin \alpha-Y \sin \mu-m g \cos \gamma), \\
\dot{\alpha}= & q+\frac{1}{m V}(m g \cos \gamma \cos \mu-L-T \sin \alpha), \\
\dot{\mu}= & p \cos \alpha+r \sin \alpha \\
& +\frac{L \tan \gamma \sin \mu+Y \tan \gamma \cos \mu+T \sin \alpha \tan \gamma \sin \mu}{m V}, \\
\dot{p}= & \frac{I_{z z}}{I_{x x} I_{z z}-I_{x z}^{2}}\left[M_{x}-\left(I_{z z}-I_{y y}\right) q r+I_{x z} q p\right] \\
& +\frac{I_{x z}}{I_{x x} I_{z z}-I_{x z}{ }^{2}}\left[M_{z}+\left(I_{x x}-I_{y y}\right) q p-I_{x z} q r\right], \\
\dot{q}= & \frac{1}{I_{y y}}\left[M_{y}-\left(I_{x x}-I_{z z}\right) p r-I_{x z}\left(p^{2}-r^{2}\right)\right], \\
\dot{r}= & \frac{I_{x z}}{I_{x x} I_{z z}-I_{x z}^{2}}\left[M_{x}+\left(I_{y y}-I_{z z}\right) q r+I_{x z} q p\right] \\
& +\frac{I_{x x}}{I_{x x} I_{z z}-I_{x z}{ }^{2}}\left[M_{z}+\left(I_{x x}-I_{y y}\right) q p-I_{x z} q r\right], \\
\ddot{\eta}_{i}+ & 2 \zeta_{i} \omega_{i} \dot{\eta}_{i}+\omega_{i}{ }^{2} \eta_{i}=N_{i}, \quad i=1,2,3,
\end{aligned}
$$

where, $x$ and $y$ are the forward position and lateral position, respectively, and $h$ is the altitude, where, $V$ is the velocity, $\chi$ is the heading angle, $\gamma$ is the flight path angel, $\mu$ is the bank angle, $p, q$ and $r$ represent the rolling rate, pitching rate and yawing rate, respectively, $g$ is the gravity acceleration, $m$ is the mass of the aircraft, $I_{x x}, I_{y y}, I_{z z}$ and $I_{x z}$ are the moment of inertia, and $M_{x}, M_{y}$ and $M_{z}$ are the rolling moment, pitching moment, and yawing moment, respectively. Since the first few flexible modes play an important role in flexible dynamics and rigid/flexible coupling, the first three flexible modes are only considered in the vehicle model. ${ }^{9,10,16)}$ The flexible state is $\left\{\eta_{1}, \dot{\eta}_{1}, \eta_{2}, \dot{\eta}_{2}, \eta_{3}, \dot{\eta}_{3}\right\}$. We have a total of five control inputs, which are elevon deflection $\delta_{e 1}, \delta_{e 2}$, rudder deflection $\delta_{r 1}, \delta_{r 2}$ and the engine throttle $\phi$. The control inputs and the rigid/flexible mode coupling will appear explicitly in the forces and moments $L, D, Y, T, M_{x}, M_{y}$, $M_{z}, N_{1}, N_{2}, N_{3}$, which are calculated using the equations mentioned in the above sections. Then, the differential equations (Eq. (11)), the forces, moments and thrust build a physics-based model of the three-dimensional vehicle.

\section{Curve-Fit Modeling}

The model in Section 4 captures interactions among the aerodynamics, structural dynamics, and propulsion of the hypersonic vehicle, and the strong couplings between the rigid and flexible modes in the model result in intractable systems for nonlinear control design. A simplified and control-oriented model (COM) can be obtained by replacing the complex force and moment functions with curve-fitted approximations. ${ }^{8,9)}$ It is a tradeoff between obtaining a model that is simple enough to facilitate nonlinear control design and capture the inherent features of the physics-based model. The simplification of the vehicle model mainly includes three 
steps: (1) data collection, (2) the curve-fitting of forces and moments, and (3) model validation.

\subsection{Data collection}

The aim of dada collection is to decide the most relevant variables for fitting, and to obtain aerodynamic values according to the intricate expressions of the forces and moments in the physics-based model presented in Section 4. The functions of forces and moments can be given by

$$
\begin{aligned}
L & \approx \bar{q} S C_{L}\left(M a, \phi, \alpha, \delta_{e 1}, \delta_{e 2}, \delta_{r 1}, \delta_{r 2}, \Delta \tau_{f}, \Delta \tau_{a}\right), \\
D & \approx \bar{q} S C_{D}\left(M a, \phi, \alpha, \delta_{e 1}, \delta_{e 2}, \delta_{r 1}, \delta_{r 2}, \Delta \tau_{f}, \Delta \tau_{a}\right), \\
Y & \approx \bar{q} S C_{Y}\left(M a, \phi, \alpha, \delta_{e 1}, \delta_{e 2}, \delta_{r 1}, \delta_{r 2}, \Delta \tau_{f}, \Delta \tau_{a}\right), \\
T & \approx \bar{q} S C_{T}\left(M a, \alpha, \phi, \Delta \tau_{f}\right), \\
M_{x} & \approx \bar{q} \bar{c} S C_{M_{x}}\left(M a, \alpha, \phi, \delta_{e 1}, \delta_{e 2}, \delta_{r 1}, \delta_{r 2}, \Delta \tau_{f}, \Delta \tau_{a}\right), \\
M_{y} & \approx z_{T} T+\bar{q} \bar{c} S C_{M_{y}}\left(M a, \alpha, \phi, \delta_{e 1}, \delta_{e 2}, \delta_{r 1}, \delta_{r 2}, \Delta \tau_{f}, \Delta \tau_{a}\right), \\
M_{z} & \approx \bar{q} \bar{c} S C_{M_{z}}\left(M a, \alpha, \phi, \delta_{e 1}, \delta_{e 2}, \delta_{r 1}, \delta_{r 2}, \Delta \tau_{f}, \Delta \tau_{a}\right), \\
N_{i} & \approx \bar{q} S C_{N_{i}}\left(M a, \alpha, \delta_{e 1}, \delta_{e 2}, \delta_{r 1}, \delta_{r 2}, \Delta \tau_{f}, \Delta \tau_{a}\right),
\end{aligned}
$$$$
i=1,2,3 \text {, }
$$

where, the reference area $S$ and mean aerodynamic chord $\bar{c}$ are constants, the aerodynamic forces are mainly related to dynamic pressure $\bar{q}$, altitude $h$, angle-of-attack $\alpha$, elevon deflection $\delta_{e 1}, \delta_{e 2}$, rudder deflection $\delta_{r 1}, \delta_{r 2}$, body deflection $\Delta \tau_{f}, \Delta \tau_{a}$, and the throttle $\phi$. The additional pitch moment generated by the thrust is added to the total pitch moment, and the thrust only includes the forebody deflection $\Delta \tau_{f}$, as the aftbody deflection $\Delta \tau_{a}$ does not affect the thrust at all. The range of values for each variable is shown in Table 1 .

\subsection{Curve-fitting}

By applying the Multivariate Stepwise Regression method, the force and moment coefficients are fitted based on the aerodynamic values. The fitting polynomials are given by

$$
\begin{aligned}
C_{L}= & C_{L}^{M a} M a+C_{L}^{\alpha} \alpha+C_{L}^{\alpha^{2}} \alpha^{2}+C_{L}^{\alpha^{2} M a} \alpha^{2} M a+C_{L}^{\delta_{e 1}} \delta_{e 1} \\
& +C_{L}^{\delta_{e 2}} \delta_{e 2}+C_{L}^{\Delta \tau_{f}} \Delta \tau_{f}+C_{L}^{\Delta \tau_{a}} \Delta \tau_{a}+C_{L}^{0}, \\
C_{D}= & C_{D}^{M a} M a+C_{D}^{\alpha} \alpha+C_{D}^{\alpha \delta_{e 1}} \alpha \delta_{e 1}+C_{D}^{\alpha \delta_{e 2}} \alpha \delta_{e 2}+C_{D}^{\delta_{e 1}^{2}} \delta_{e 1}^{2} \\
& +C_{D}^{\delta_{e 2}^{2}} \delta_{e 2}^{2}+C_{D}^{\delta_{r 1}} \delta_{r 1}+C_{D}^{\delta_{11}^{2}} \delta_{r 1}^{2}+C_{D}^{\delta_{r 2}^{2}} \delta_{r 2}^{2} \\
& +C_{D}^{\left(\Delta \tau_{f}+\alpha\right)}\left(\Delta \tau_{f}+\alpha\right)+C_{D}^{\left(\Delta \tau_{f}+\alpha\right)^{2}}\left(\Delta \tau_{f}+\alpha\right)^{2} \\
& +C_{D}^{\Delta \tau_{a}} \Delta \tau_{a}+C_{D}^{0}, \\
C_{Y}= & C_{Y}^{\alpha \delta_{r 1}} \alpha \delta_{r 1}+C_{Y}^{\alpha \delta_{r 2}} \alpha \delta_{r 2}+C_{Y}^{\delta_{r 1}^{2}} \delta_{r 1}^{2}+C_{Y}^{\delta_{r 2}^{2}} \delta_{r 2}^{2}+C_{Y}^{0}, \\
C_{T}= & C_{T}^{M a \cdot \phi} M a \cdot \phi+C_{T}^{M a \cdot \alpha \cdot \phi} M a \cdot \alpha \cdot \phi+C_{T}^{\alpha} \alpha+C_{T}^{\phi} \phi \\
& +C_{T}^{\phi \alpha} \phi \alpha+C_{D}^{\Delta \tau_{f}} \Delta \tau_{f}+C_{D}^{\Delta \tau_{f} \phi} \Delta \tau_{f} \phi \\
& +C_{D}^{\Delta \tau_{f} \phi \alpha} \Delta \tau_{f} \phi \alpha+C_{T}^{0}, \\
C_{M_{x}}= & C_{M_{x}}^{\delta_{e 1}} \delta_{e 1}+C_{M_{x}}^{\delta_{e 2}} \delta_{e 2}+C_{M_{x}}^{0},
\end{aligned}
$$

Table 1. Range of the variables.

\begin{tabular}{lc|ll}
\hline Variable & Range & Variable & Range \\
\hline$V(\mathrm{~m} / \mathrm{s})$ & $2286-3353$ & $\delta_{r 1}\left(^{\circ}\right)$ & $-6-6$ \\
$\alpha\left(^{\circ}\right)$ & $-5-5$ & $\delta_{r 2}\left(^{\circ}\right)$ & $-6-6$ \\
$\phi$ & $0.05-1.5$ & $\Delta \tau_{f}\left(^{\circ}\right)$ & $-2-2$ \\
$\delta_{e 1}\left(^{\circ}\right)$ & $-10-10$ & $\Delta \tau_{a}\left(^{\circ}\right)$ & $-2-2$ \\
$\delta_{e 2}\left({ }^{\circ}\right)$ & $-10-10$ & & \\
\hline
\end{tabular}

$$
\begin{aligned}
C_{M_{y}}= & C_{M_{y}}^{M a} M a+C_{M_{y}}^{\alpha a} \alpha+C_{M_{y}}^{\phi} \phi+C_{M_{y}}^{\alpha^{2}} \alpha^{2}+C_{M_{y}}^{\delta_{e 1}} \delta_{e 1} \\
& +C_{M_{y}}^{\delta_{e 2}} \delta_{e 2}+C_{M_{y}}^{\delta_{r 1}} \delta_{r 1}+C_{M_{y}}^{\alpha M a \Delta \tau_{f}} \alpha M a \Delta \tau_{f} \\
& +C_{M_{y}}^{\left(\Delta \tau_{f}+\alpha\right)^{2} M a}\left(\Delta \tau_{f}+\alpha\right)^{2} M a+C_{M_{y}}^{\left(\Delta \tau_{f}\right)^{3}}\left(\Delta \tau_{f}\right)^{3} \\
& +C_{M_{y}}^{\left(\Delta \tau_{f}\right)^{3} M a}\left(\Delta \tau_{f}\right)^{3} M a+C_{M_{y}}^{\Delta \tau_{a}} \Delta \tau_{a}+C_{M_{y}}^{0}, \\
C_{M_{z}}= & C_{M_{z}}^{M a} M a+C_{M_{z}}^{\alpha} \alpha+C_{M_{z}}^{M a \alpha} M a \alpha+C_{M_{z}}^{\alpha^{2}} \alpha^{2}+C_{M_{z}}^{\alpha \delta_{e 1}} \alpha \delta_{e 1} \\
& +C_{M_{z}}^{\delta_{e 1}^{2}} \delta_{e 1}^{2}+C_{M_{z}}^{\alpha \delta_{e 2}} \alpha \delta_{e 2}+C_{M_{z}}^{\delta_{e 2}^{2}} \delta_{e 2}^{2}+C_{M_{z}}^{\delta_{r 1}^{2}} \delta_{r 1}^{2} \\
& +C_{M_{z}}^{\alpha \delta_{r 1}} \alpha \delta_{r 1}+C_{M_{z}}^{\delta_{r 2}^{2}} \delta_{r 2}^{2}+C_{M_{z}}^{\alpha \delta_{r 2}^{2}} \alpha \delta_{r 2}^{2}+C_{M_{z}}^{\delta_{r 1}^{2} M a} \delta_{r 1}^{2} M a \\
& +C_{M_{z}}^{\delta_{e 1} \Delta \tau_{f}} \delta_{e 1} \Delta \tau_{f}+C_{M_{z}}^{\delta_{e 2} \Delta \tau_{f}} \delta_{e 2} \Delta \tau_{f}+C_{M_{z}}^{\delta_{r 1}^{3}} \delta_{r 1}^{3}+C_{M_{z}}^{0}, \\
C_{N_{i}}= & C_{N_{i}}^{\alpha} \alpha+C_{N_{i}}^{\delta_{e 1}} \delta_{e 1}+C_{N_{i}}^{\delta_{e 2}} \delta_{e 2}+C_{N_{i}}^{\delta_{r 1}} \delta_{r 1}+C_{N_{i}}^{\delta_{r 2}} \delta_{r 2} \\
& +C_{N_{i}}^{\Delta \tau_{f}} \Delta \tau_{f}+C_{N_{i}}^{\Delta \tau_{a}} \Delta \tau_{a}+C_{N_{i}}^{0}, \quad i=1,2,3 .
\end{aligned}
$$

The coefficients are all the functions of rigid states, flexible modes, and control inputs. The forms of the polynomialbased functions are decided by the plots of the aerodynamic values, and attempt to capture the inherent couplings among the aerodynamic, flexible structure and propulsion system. The functions of the rolling moment and the generalized force coefficients contain linear terms with relevant variables, and the cross-terms and higher-order terms with respect to variables are concluded in the others. The variables that contribute little to accuracy are neglected, and the fitting progress is a compromise between model complexity and fitting precision.

\subsection{Model validation}

The model validation is mainly to check whether or not the curve-fitting polynomials are consistent with the aerodynamic values. This can be compared using three-dimensional plots and fitting errors. We firstly examine the three-dimensional plots of the aerodynamic forces and moments with variables under certain flight conditions. Figures 4-7 show the trends of lift, drag, pitching moment and thrust with relevant variables, respectively. We can see that the trend of the fit curve and the plots of aerodynamic values is approximated. Tables 2-5 list the fitting results and fitting values of the forces and moments. It is clear that the $R$-squared and the mean squared errors (RSEs) meet precision requirements. The $p$-value shows that the basic functions are very significant in the fitting. We can also see that deviations exist in the fitting results and robust control design is needed to stabilize the vehicle model. 


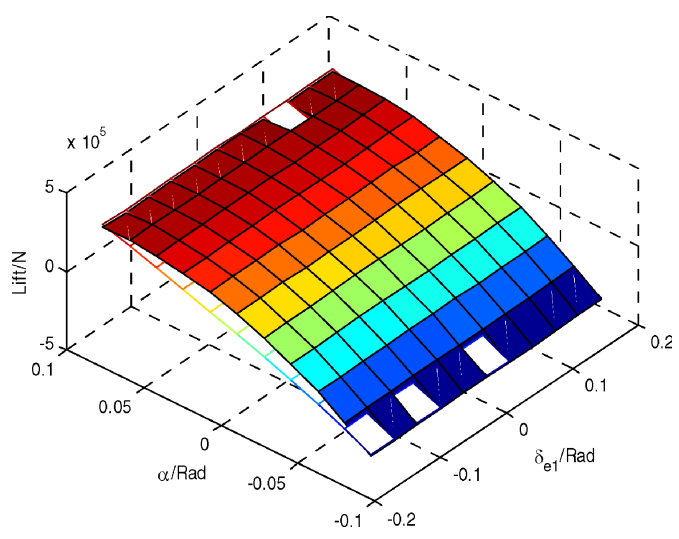

Fig. 4. Lift versus $\alpha$ and $\Delta \delta_{e 1}$.

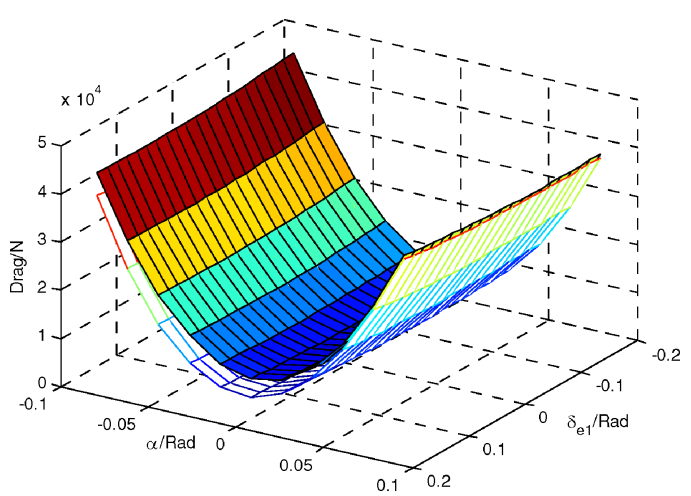

Fig. 5. Drag versus $\alpha$ and $\delta_{e 1}$.

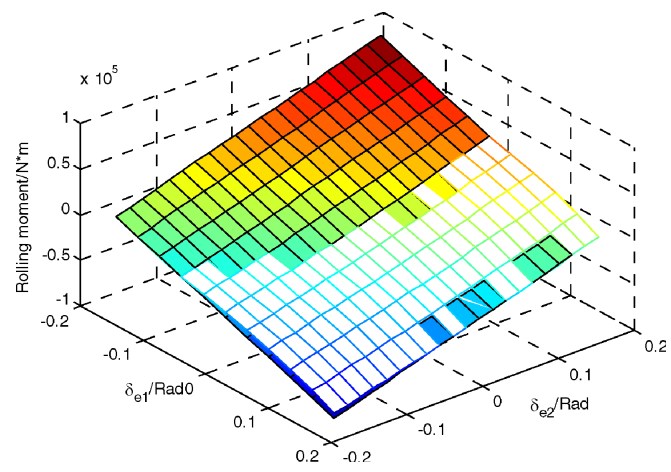

Fig. 6. Rolling moment versus $\delta_{e 1}$ and $\delta_{e 2}$.

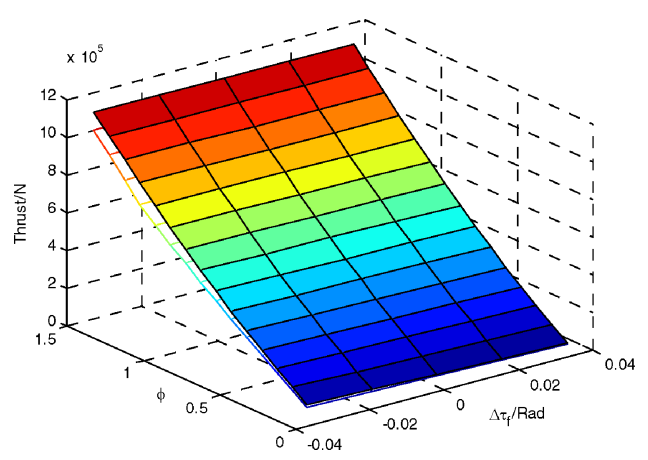

Fig. 7. Thrust versus $\phi$ and $\Delta \tau_{f}$.
Table 2. The fitting results.

\begin{tabular}{lllc}
\hline \multicolumn{1}{c}{ Force coefficients } & $R$-squared & \multicolumn{1}{c}{ RSE } & $p$-value \\
\hline Lift & 0.853828 & 0.00975397 & 0 \\
Drag & 0.943842 & 0.00025164 & 0 \\
Side force & 0.98888 & $9.87 \times 10^{-7}$ & 0 \\
Thrust & 0.97892 & 38.4937 & 0 \\
Rolling moment & 0.965534 & $4.09 \times 10^{-6}$ & 0 \\
Pitching moment & 0.812444 & 0.0299675 & 0 \\
Yawing moment & 0.826789 & $4.045 \times 10^{-3}$ & 0 \\
1st generalized force & 0.941424 & 0.0274843 & 0 \\
2nd generalized force & 0.931114 & 0.600195 & 0 \\
3rd generalized force & 0.919984 & 5.67826 & 0 \\
\hline
\end{tabular}

Table 3. Fitting values of thrust.

\begin{tabular}{lc|lc}
\hline Terms & Values & Terms & Values \\
\hline$C_{T}^{M a \cdot \phi}$ & -77.7485 & $C_{T}^{\Delta \tau_{f}}$ & -228.55 \\
$C_{T}^{M a \cdot \alpha \phi}$ & 703.97 & $C_{T}^{\Delta \tau_{f} \cdot \phi}$ & 122.087 \\
$C_{T}^{\alpha}$ & -138.071 & $C_{T}^{\Delta \tau_{f} \cdot \phi \cdot \alpha}$ & 698.791 \\
$C_{T}^{\phi}$ & 962.394 & $C_{T}^{0}$ & 18.2222 \\
$C_{T}^{\alpha \phi}$ & -9366.01 & & \\
\hline
\end{tabular}

Table 4. Fitting values of generalized forces

\begin{tabular}{cc|cc}
\hline Terms & Values & Terms & Values \\
\hline$C_{N_{1}}^{\alpha}$ & 1081.37 & $C_{N_{2}}^{\delta_{r 2}}$ & 5419.93 \\
$C_{N_{1}}^{\delta_{e 1}}$ & 320.924 & $C_{N_{2}}^{\Delta \tau_{f}}$ & 16167.9 \\
$C_{N_{1}}^{\delta_{e 2}}$ & 320.924 & $C_{N_{2}}^{\Delta \tau_{a}}$ & -15659.1 \\
$C_{N_{1}}^{\delta_{11}}$ & -259.387 & $C_{N_{2}}^{0}$ & -413.892 \\
$C_{N_{1}}^{\delta_{2}}$ & 259.387 & $C_{N_{3}}^{\alpha}$ & 188067 \\
$C_{N_{1}}^{\Delta \tau_{f}}$ & 845.114 & $C_{N_{3}}^{\delta_{e 1}}$ & 56071.7 \\
$C_{N_{1}}^{\Delta \tau_{a}}$ & -749.415 & $C_{N_{3}}^{\delta_{e 2}}$ & 56071.7 \\
$C_{N_{1}}^{0}$ & -20.9317 & $C_{N_{3}}^{\delta_{r 1}}$ & -45320.1 \\
$C_{N_{2}}^{\alpha}$ & 20824.5 & $C_{N_{3}}^{\delta_{r 2}}$ & 45320.1 \\
$C_{N_{2}}^{\delta_{e 1}}$ & 6705.74 & $C_{N_{3}}^{\Delta \tau_{f}}$ & 154851 \\
$C_{N_{2}}^{\delta_{2}}$ & 6705.74 & $C_{N_{3}}^{\Delta \tau_{a}}$ & -130938 \\
$C_{N_{2}}^{\delta_{r 1}}$ & -5419.93 & $C_{N_{3}}^{0}$ & -3814.82 \\
\hline
\end{tabular}

\section{Dynamic Analysis}

\subsection{Rigid/Flexible modes}

Using the 6-DOF curve-fitting model, it is possible to characterize the flight dynamics of the vehicle. The aircraft is trimmed in steady level flight at an altitude of $25,908 \mathrm{~m}$ and Mach 8. The flexible vehicle model is then linearized at the trim states. The eigenvalues of the system are given in Table 6. It can be seen that the vehicle has an unstable shortperiod mode, a stable Alt/Phugoid mode, a stable velocity mode and three stable flexible modes. The lateral dynamics feature an unstable spiral mode and an unstable dutch roll mode. Additionally, the rigid states are coupled with the flexible modes. The dynamics analysis shows that the flexible vehicle model is unstable both longitudinally and laterally, and a robust control design is required to maintain the aircraft stability. 
Table 5. Fitting values of the aerodynamic.

\begin{tabular}{|c|c|c|c|}
\hline Terms & Values & Terms & Values \\
\hline$C_{L}^{M a}$ & 0.0178046 & $C_{M_{y}}^{M a}$ & -0.0250006 \\
\hline$C_{L}^{\alpha}$ & 0.143344 & $C_{M_{y}}^{\alpha}$ & -0.0433775 \\
\hline$C_{L}^{\alpha^{2}}$ & 18.9777 & $C_{M_{y}}^{\phi}$ & -0.0146508 \\
\hline$C_{L}^{\alpha^{2} M a}$ & -2.3008 & $C_{M_{y}}^{\alpha^{2}}$ & -18.4042 \\
\hline$C_{L}^{\delta_{e 1}}$ & 0.00109479 & $C_{M_{y}}^{\delta_{e 1}}$ & 0.000352029 \\
\hline$C_{L}^{\delta_{e 2}}$ & 0.00109479 & $C_{M_{y}}^{\delta_{e 2}}$ & 0.000352029 \\
\hline$C_{L}^{\Delta \tau_{f}}$ & 0.145858 & $C_{M_{y}}^{\delta_{r 1}}$ & $3.16288 \times 10^{-5}$ \\
\hline$C_{L}^{\Delta \tau_{a}}$ & -0.0025468 & $C_{M_{y}}^{\alpha M a \Delta \tau_{f}}$ & 5.16642 \\
\hline$C_{L}^{0}$ & -0.148247 & $C_{M_{y}}^{\left(\Delta \tau_{f}+\alpha\right)^{2}}$ & 2.61794 \\
\hline$C_{D}^{M a}$ & $-5.58025 \times 10^{-5}$ & $C_{M_{y}}^{\left(\Delta \tau_{f}\right)^{3}}$ & -236.712 \\
\hline$C_{D}^{\alpha}$ & 0.00324985 & $C_{M_{y}}^{\left(\Delta \tau_{f}\right)^{3} M a}$ & 17.3169 \\
\hline$C_{D}^{\alpha \delta_{e 1}}$ & 0.00267406 & $C_{M_{y}}^{\Delta \tau_{a}}$ & 0.00155477 \\
\hline$C_{D}^{\alpha \delta_{e 2}}$ & 0.00267406 & $C_{M_{y}}^{0}$ & 0.15237 \\
\hline$C_{D}^{\delta_{e 1}^{2}}$ & 0.00121896 & $C_{M_{z}}^{M a}$ & $-2.1189 \times 10^{-7}$ \\
\hline$C_{D}^{\delta_{e 2}^{2}}$ & 0.00121896 & $C_{M_{z}}^{\alpha}$ & $4.45623 \times 10^{-5}$ \\
\hline$C_{D}^{\delta_{r 1}}$ & 0.000901307 & $C_{M_{z}}^{M a \alpha}$ & $-1.16004 \times 10^{-6}$ \\
\hline$C_{D}^{\delta^{2}}$ & 0.00144168 & $C_{M_{z}}^{\alpha^{2}}$ & $4.2015 \times 10^{-5}$ \\
\hline$C_{D}^{\delta_{r 2}^{2}}$ & 0.00716517 & $C_{M_{z}}^{\alpha \delta_{e 1}}$ & 0.0001485 \\
\hline$C_{D}^{\Delta \tau_{f}+\alpha}$ & 0.00434444 & $C_{M_{z}}^{\delta_{e 1}^{2}}$ & 0.000108149 \\
\hline$C_{D}^{\left(\Delta \tau_{f}+\alpha\right)^{2}}$ & 0.186238 & $C_{M_{z}}^{\alpha \delta_{e 2}}$ & -0.0001485 \\
\hline$C_{D}^{\Delta \tau_{a}}$ & 0.00223723 & $C_{M_{z}}^{\delta_{e 2}^{2}}$ & -0.00010815 \\
\hline$C_{D}^{0}$ & 0.0007666681 & $C_{M_{z}}^{\delta_{r 1}^{2}}$ & -0.00002185 \\
\hline$C_{Y}^{\alpha \delta_{r 1}}$ & 0.000758573 & $C_{M_{z}}^{\alpha \delta_{r 1}}$ & 0.000241564 \\
\hline$C_{Y}^{\alpha \delta_{r 2}}$ & 0.000758573 & $C_{M_{z}}^{\delta_{r 2}^{2}}$ & 0.000637889 \\
\hline$C_{Y}^{\alpha \delta_{r 1}^{2}}$ & 0.000906997 & $C_{M_{z}}^{\delta_{r 2}^{2} \alpha}$ & -0.00120894 \\
\hline$C_{Y}^{\alpha \delta_{r 2}^{2}}$ & -0.000906997 & $C_{M_{z}}^{\delta_{r 1}^{2} M a}$ & -0.00027677 \\
\hline$C_{Y}^{0}$ & 0.000000021 & $C_{M_{z}}^{\delta_{e 1} \Delta \tau_{f}}$ & -0.00025495 \\
\hline$C_{M_{x}}^{\delta_{e 1}}$ & -0.000107341 & $C_{M_{z}}^{\delta_{e 2} \Delta \tau_{f}}$ & 0.000254945 \\
\hline$C_{M_{x}}^{\delta_{e 2}}$ & 0.000107341 & $C_{M_{z}}^{\delta_{r 1}^{r^{*}}}$ & 0.00668575 \\
\hline$C_{M_{x}}^{0}$ & $-9.40556 \times 10^{-8}$ & $C_{M_{z}}^{0}$ & $1.8391 \times 10^{-6}$ \\
\hline
\end{tabular}

Table 6. Eigenvalues of the vehicle model.

\begin{tabular}{cccc}
\hline \multicolumn{1}{c}{ Eigenvalues } & Damping ratio & Frequency & Mode \\
\hline $0.0216 \pm 0.0529 i$ & -0.377 & 0.0529 & Short-period \\
$-0.0442 \pm 0.0279 i$ & 0.345 & 0.0279 & Alt/phugoid \\
$-1.94 \times 10^{-13}$ & 1 & $-1.94 \times 10^{-13}$ & Velocity \\
0.0505 & -1 & 0.0515 & Spiral \\
$0.5285 \pm 23.7020 i$ & -0.0223 & 23.7 & Dutch roll \\
$-0.3190 \pm 20.6088 i$ & 0.0209 & 20.6088 & 1st aeroelastic \\
$-0.9938 \pm 50.1218 i$ & 0.0198 & 50.1218 & 2nd aeroelastic \\
$-1.9767 \pm 99.2858 i$ & 0.0199 & 99.2858 & 3rd aeroelastic \\
\hline
\end{tabular}

\section{2. $\quad$ Rigid/Flexible coupling}

The aerodynamic, flexible structure dynamic and propulsion system interact with each other, which results in strong coupling effects in the model. In order to analyze the influence of flexible effects on the dynamic characteristics of aircraft, Figures 8-17 present the Bode plots of the control inputs versus the rigid states selected. Figures $8-15$ show the Bode plots of the elevon deflections and rudder deflections as compared to the altitude and flight path angle. It is clear that fluctuations occur as the flexible mode appears, and the model contains the coupling between the rigid and flexible dynamics.

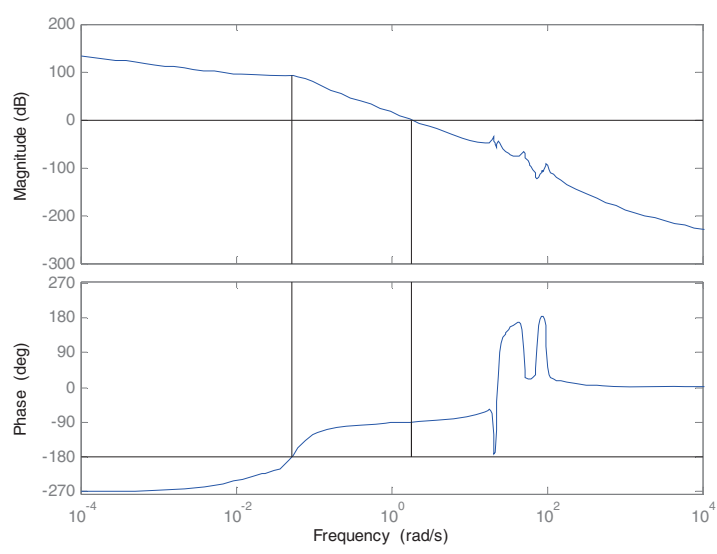

Fig. 8. The bode graph of altitude $(h)$ on $\delta_{e 1}$.

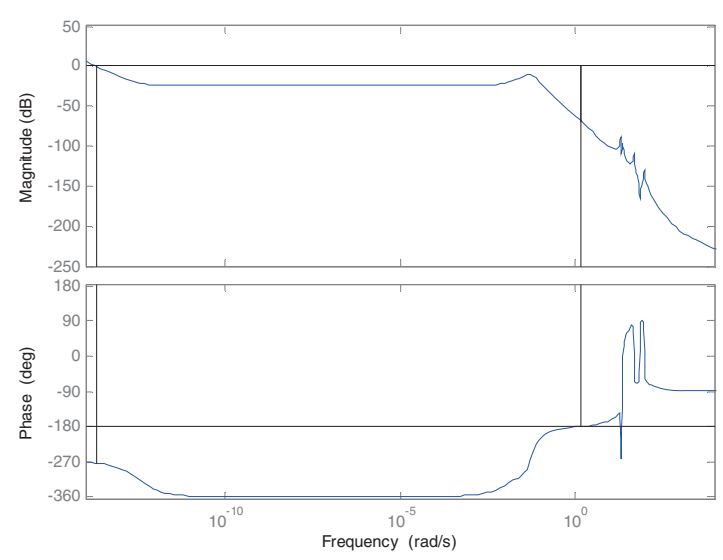

Fig. 9. The bode graph of flight path angle $(\gamma)$ on $\delta_{e 1}$.

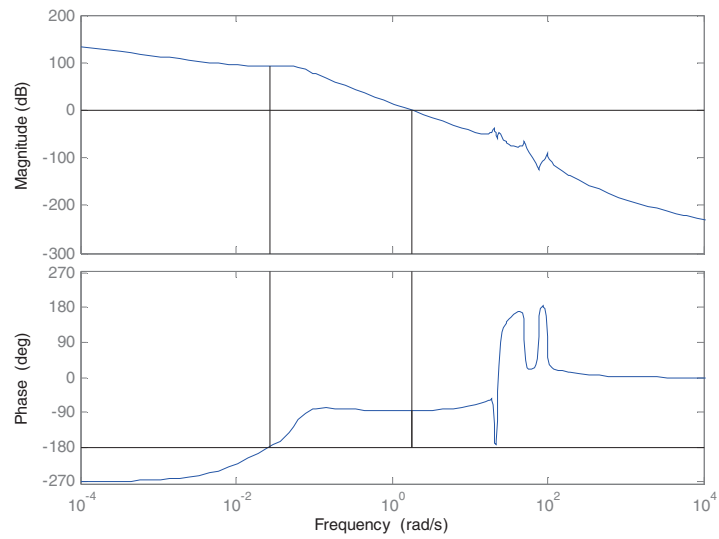

Fig. 10. The bode graph of the altitude $(h)$ on $\delta_{e 2}$.

Figures 16 and 17 show the Bode plots of the engine throttle as compared to the altitude and flight path angle. It is also clear that fluctuations occur when the flexible mode appears at the lower frequency, and the model contains the effects of the airframe, structure and propulsion interactions. From these results, it is apparent that a control scheme is required to suppress the flexible mode at the time of structural damage. 
Trans. Japan Soc. Aero. Space Sci., Vol. 63, No. 4, 2020

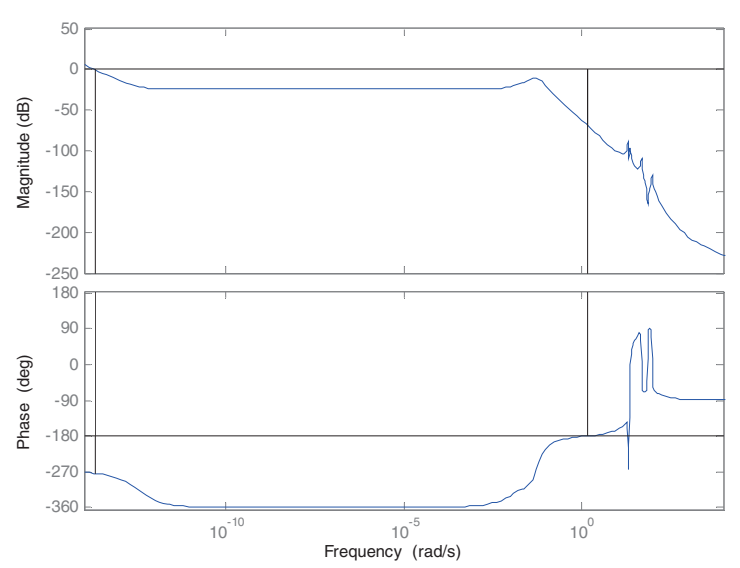

Fig. 11. The bode graph of the flight path angle $(\gamma)$ on $\delta_{e 2}$.

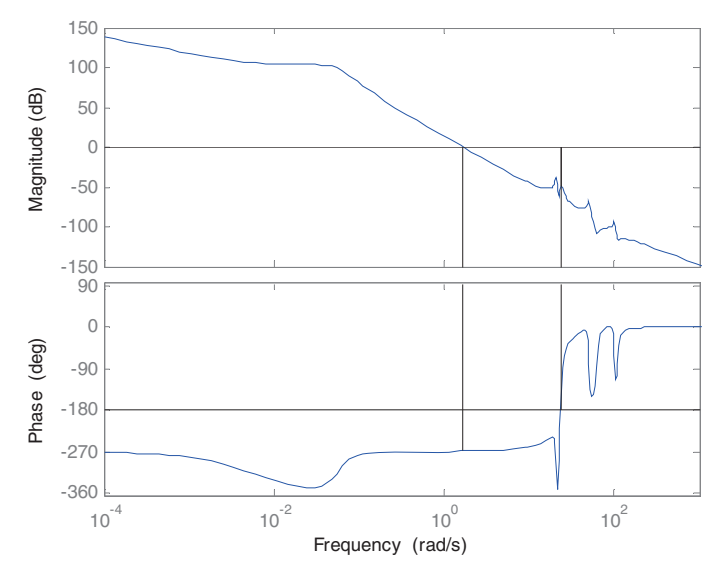

Fig. 12. The bode graph of the altitude $(h)$ on $\delta_{r 1}$.

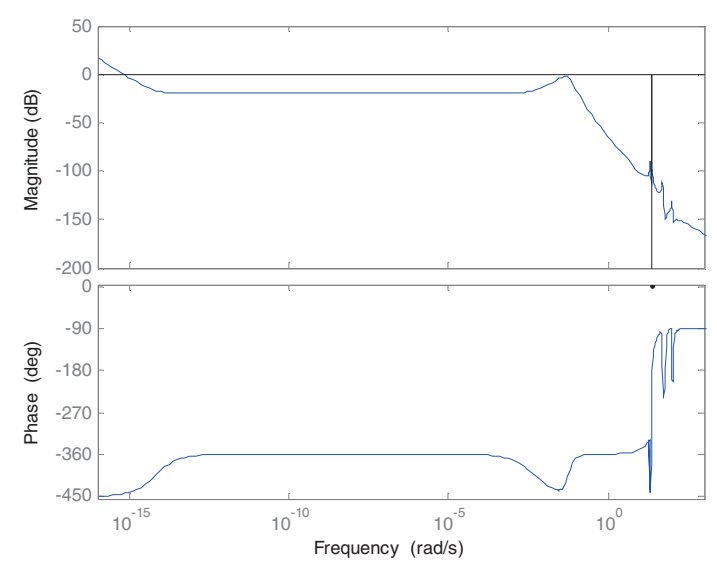

Fig. 13. The bode graph of the flight path angle $(\gamma)$ on $\delta_{r 1}$.

\section{Conclusion}

Based on a three-dimensional flexible hypersonic flight vehicle, this paper firstly uses the mechanism analysis method to estimate the aerodynamic forces and moments, which contain the flexible bending of the forebody and aftbody. A physics-based vehicle model with heave couplings is developed. Then, the Multivariate stepwise regression method is used to find suitable curve-fit approximations of forces and moments, and a control-oriented 6-DOF vehicle model with

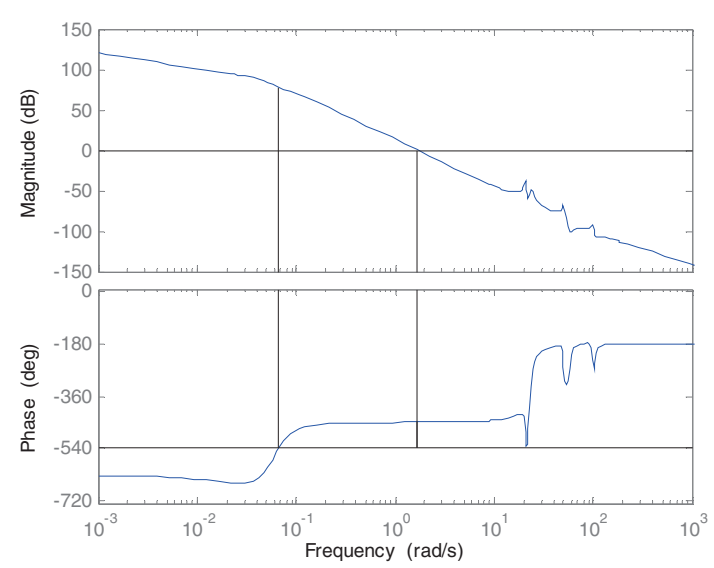

Fig. 14. The bode graph of the altitude $(h)$ on $\delta_{r 2}$.

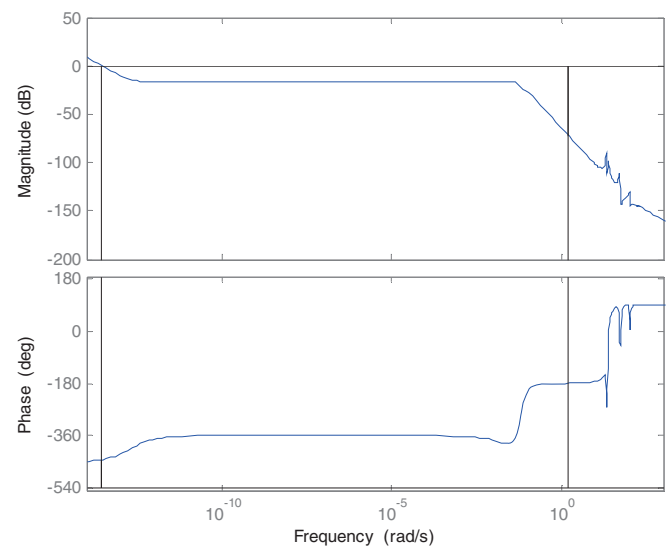

Fig. 15. The bode graph of the flight path angle $(\gamma)$ on $\delta_{r 2}$.

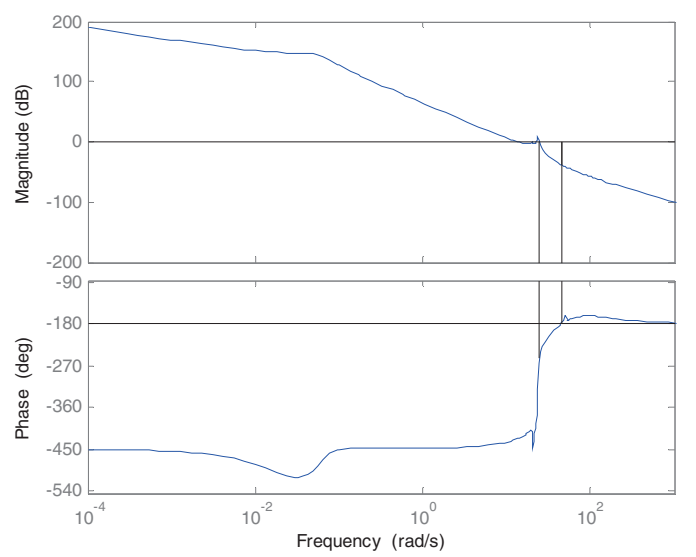

Fig. 16. The bode graph of the altitude $(h)$ on $\phi$.

aerodynamic, structure and propulsion interactions is established. The results show that the COM reduces the complexity of the physics-based model, meets the accuracy requirements, and retains the inherent rigid/flexible couplings. The simulation framework developed for this work will support the design of hypersonic vehicle control systems. In the future, a multi-disciplinary modeling method and robust control system design for the vehicle model will be studied. 


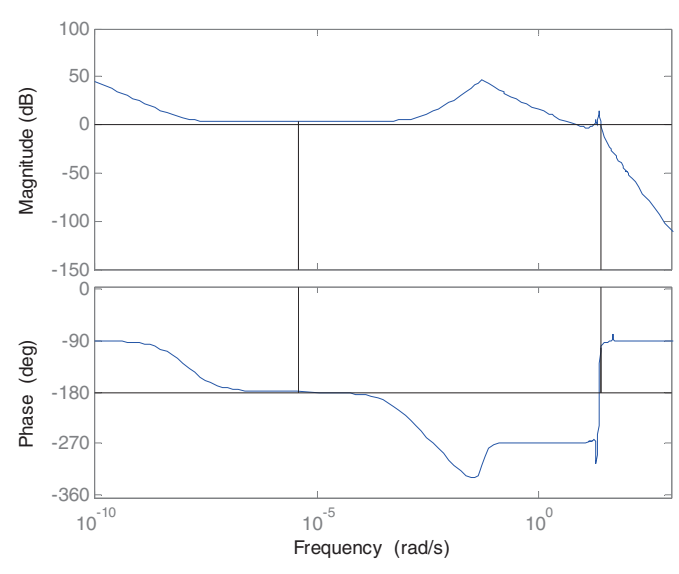

Fig. 17. The bode graph of the flight path angle $(\gamma)$ on $\phi$.

\section{Acknowledgments}

This work was supported by the Mathematics Tian Yuan Fund of the National Natural Science Foundation Committee (11426164), the Fundamental Research Funds for the Universities of Tianjin (2018KJ112), and Tianjin Municipal Science of Technology Commission, Grant No. 19JCYBJC22000.

\section{References}

1) Mcnamara, J. J. and Friedmann, P. P.: Aeroelastic and Aerothermoelastic Analysis of Hypersonic Vehicles: Current Status and Future Trends, The 48th AIAA/ASME/ASCE/ AHS/ASC Structures, Structural Dynamics, and Materials Conference, Hawaii, USA, 2007. https://doi.org/10.2514/6.2007-2013

2) Chavez, F. R. and Schmidt, D. K.: Analytical Aeropropulsive/Aeroelastic Hypersonic Vehicle Model with Dynamic Analysis, J. Guid. Control Dynam., 17, 6 (1994), pp. 1308-1319.

3) Bolender, M. A. and Doman, D. B.: Nonlinear Longitudinal Dynamical Model of an Air-Breathing Hypersonic Vehicle, J. Spacecraft Rockets, 44, 2 (2007), pp. 374-386.

4) Bolender, M. A., Oppenheimer, M. W., and Doman, D. B.: Effects of Unsteady and Viscous Aerodynamics on the Dynamics of a Flexible Air-Breathing Hypersonic Vehicle, AIAA Atmospheric Flight Mechanics Conference and Exhibit, Hilton Head, USA, 2007. https: //doi.org/10.2514/6.2007-6397

5) Oppenheimer, M. W., Skujins, T., Doman, D. B., and Bolender, M. A.: A Flexible Hypersonic Vehicle Model Developed with Piston Theory, AIAA Atmospheric Flight Mechanics Conference and Exhibit, Hilton Head, USA, 2007. https://doi.org/10.2514/6.2007-6396

6) Oppenheimer, M. W., Doman, D. B., McNamara, J. J., and Culle, A. J.: Viscous Effects for a Hypersonic Vehicle Model, AIAA Atmospheric Flight Mechanics Conference and Exhibit, Hawaii, USA, 2008. https: //doi.org/10.2514/6.2008-6382

7) Williams, T., Bolender, M. A., Doman, D. B., and Morataya, O.: An Aerothermal Flexible Mode Analysis of a Hypersonic Vehicle, AIAA Atmospheric Flight Mechanics Conference and Exhibit, Keystone, Colorado, USA, 2006. https://doi.org/10.2514/6.2006-6647

8) Parker, J. T., Serrani, A., Yurkovich, S., Bolender, M. A., and Doman, D. B.: Control-Oriented Modeling of an Air-Breathing Hypersonic Vehicle, J. Guid. Control Dynam., 30, 3 (2007), pp. 402-406.

9) Sigthorsson, D. O. and Serrani, A.: Development of Linear ParameterVarying Models of Hypersonic, Air-Breathing Vehicles, AIAA Guidance, Navigation, and Control Conference, Chicago, USA, 2009. https://doi.org/10.2514/6.2009-6282

10) Zhang, X. B. and Zong, Q.: Modeling and Analysis of an Air-
Breathing Flexible Hypersonic Vehicle, Math. Problem Eng., 6 (2014), pp. 759-765.

11) Shen, H. D., Liu, Y. B., Chen, B. Y., and Lu, Y. P.: Control-Relevant Modeling and Performance Limitation Analysis for Flexible AirBreathing Hypersonic Vehicles, Aerospace Sci. Technol., 76 (2018), pp. 340-349.

12) Zhang, D., Tang, S., Cao, L., Cheng, F., and Deng, F.: Research on Control-Oriented Coupling Modeling for Air-breathing Hypersonic Propulsion Systems, Aerospace Sci. Technol., 84 (2019), pp. 143-157.

13) Fiorentini, L. and Serrani, A.: Adaptive Restricted Trajectory Tracking for a Non-minimum Phase Hypersonic Vehicle Model, Automatica, 48, 7 (2012), pp. 1248-1261.

14) Zong, Q., Wang, J., Tian, B. L., and Tao, Y.: Quasi-continuous Highorder Sliding Mode Controller and Observer Design for Flexible Hypersonic Vehicle, Aerospace Sci. Technol., 27, 1 (2013), pp. 127-137.

15) Fiorentini, L., Serrani, A., Bolender, M. A., and Doman, D. B.: Nonlinear Robust Adaptive Control of Flexible Air-Breathing Hypersonic Vehicles, J. Guid. Control Dynam., 32, 2 (2009), pp. 402-417.

16) Levin, J., Ioannou, P. A., and Mirmirani, M. D.: Adaptive Mode Suppression Scheme for an Aeroelastic Air-Breathing Hypersonic Cruise Vehicle, AIAA Guidance, Navigation and Control Conference and Exhibit, Honolulu, Hawaii, USA, 2008. https://doi.org/10.2514/6.20087137

17) Clark, A. D., Mirmirani, M. D., Wu, C., Choi, S., and Kuipers, M.: An Aero-Propulsion Integrated Elastic Model of a Generic Air-breathing Hypersonic Vehicle, AIAA, GNC Conference and Exhibit, Keystone, Colorado, USA, 2006. https://doi.org/10.2514/6.2006-6560

18) Clark, A. D., Wu, C., Mirmirani, M. D., Choi, S., and Kuipers, M.: Development of an Airframe-Propulsion Integrated Generic Hypersonic Vehicle Model, The 44th AIAA Aerospace Sciences Meeting and Exhibit, Nevada, USA, 2006. https://doi.org/10.2514/6.2006-218

19) Zeng, K. C., Xiang, J. W., and Li, D. C.: Aeroservoelastic Modeling and Analysis of a Canard-Configured Air-Breathing Hypersonic Vehicles, Chinese J. Aeronaut., 26, 4 (2013), pp. 831-840.

20) Keshmiri, S.: Nonlinear and Linear Longitudinal and LateralDirectional Dynamical Model of Air-Breathing Hypersonic Vehicle, 15th AIAA International Space Planes and Hypersonic Systems and Technologies Conference, Dayton, Ohio, USA, 2008. https://doi.org/ 10.2514/6.2008-2531

21) Zong, Q., You, M., Zeng, F. L., and Dou, L. Q.: Aeroservoelastic Modeling and Analysis of a Six-DOF Hypersonic Flight Vehicle, Proc. Inst. Mech. Eng. G J. Aerosp. Eng., 230, 7 (2016), pp. 1240-1251.

22) Ye, K., Ye, A. Y., Li, C. N., and Wu, J.: Effects of the Aerothermoelastic Deformation on the Performance of the Three-Dimensional Hypersonic Inlet, Aerospace Sci. Technol., 84 (2019), pp. 747-762.

23) Frendreis, S. G. V., Skujins, T., and Cesnik, C. E. S.: Six-Degree-ofFreedom Simulation of Hypersonic Vehicles, AIAA Atmospheric Flight Mechanics Conference, Chicago, Illinois, USA, 2009. https: //doi.org/10.2514/6.2009-5601

24) Frendreis, S. G. V. and Cesnik, C. E. S.: 3D Simulation of Flexible Hypersonic Vehicles, AIAA Atmospheric Flight Mechanics Conference, Toronto, Ontario, Canada, 2010. https://doi.org/10.2514/6.2010-8229

25) Sudalagunta, P. R., Sultan, C., Kapania, R. K., Watson, L. T., and Raj, P.: Aeroelastic Control-Oriented Modeling of an Airbreathing Hypersonic Vehicle, J. Guid. Control Dynam., 41, 5 (2018), pp. 1136-1149.

26) Ricketts, R., Noll, T., Whitlow, W., Jr., and Huttsell, L.: An Overview of Aeroelasticity Studies for the National Aero-Space Planes, AIAA/ ASME/ASCE/AHS/ASC Structures, Structural Dynamics, and Materials Conference, Washington, DC, USA, 1993, pp. 152-162. https: //doi.org/10.2514/6.1993-1313

27) Shakiba, M. and Serrani, A.: Control Oriented Modeling of 6-DOF Hypersonic Vehicle Dynamics, AIAA Guidance, Navigation, and Control Conference, Oregon, Portland, 2011. https://doi.org/10.2514/ 6.2011-6227 